Research Paper

\title{
Respiratory Magnetogram Detected with a MEMS Device
}

\author{
Saul M. Dominguez-Nicolas ${ }^{1}$, Raul Juarez-Aguirre ${ }^{1}$, Agustin L. Herrera-May ${ }^{1}$, Pedro Garcia-Ramirez ${ }^{1}$, \\ Eduard Figueras ${ }^{2}$, Edmundo A. Gutierrez-D. ${ }^{3}$, Jesus A. Tapia ${ }^{4}$, Argelia Trejo ${ }^{4}$ and Elias Manjarrez ${ }^{4}$ \\ 1. Research Center for Micro and Nano Technology, Universidad Veracruzana, Calzada Adolfo Ruíz Cortines 455, 94294, Boca del Río, \\ Ver., Mexico \\ 2. Microelectronics Institute of Barcelona IMB-CNM, CSIC, Bellaterra, 08193, Spain. \\ 3. Department of Electronics, Instituto Nacional de Astrofisica Optica y Electronica, INAOE, Puebla, Pue., Mexico. \\ 4. Institute of Physiology, Benemerita Universidad Autonoma de Puebla, 14 Sur 6301, Colonia San Manuel, Apartado Postal 406, CP 72570, \\ Puebla, Pue., Mexico.
}

$\triangle$ Corresponding author: elias.manjarrez@correo.buap.mx or eliasmanjarrez@gmail.com

(C) Ivyspring International Publisher. This is an open-access article distributed under the terms of the Creative Commons License (http://creativecommons.org/ licenses/by-nc-nd/3.0/). Reproduction is permitted for personal, noncommercial use, provided that the article is in whole, unmodified, and properly cited.

Received: 2012.06.12; Accepted: 2012.11.26; Published: 2013.08.27

\begin{abstract}
Magnetic fields generated by the brain or the heart are very useful in clinical diagnostics. Therefore, magnetic signals produced by other organs are also of considerable interest. Here we show first evidence that thoracic muscles can produce a strong magnetic flux density during respiratory activity, that we name respiratory magnetogram. We used a small magnetometer based on microelectromechanical systems (MEMS), which was positioned inside the open thoracic cage of anaesthetized and ventilated rats. With this new MEMS sensor of about $20 \mathrm{nT}$ resolution, we recorded a strong and rhythmic respiratory magnetogram of about $600 \mathrm{nT}$.
\end{abstract}

Key words: respiration, thoracic muscles, ribs, thoracic cage, magnetocardiogram, magnetometer.

\section{Introduction}

In 1963, with a set of copper induction coils, Baule and McFee [1] recorded for the first time a magnetocardiogram (MCG). Although this recording was very noisy, the discovery of the MCG stimulated future technological investigations. A subsequent study [2] improved the MCG by means of three mutually perpendicular induction coils, coupled by an impedance transformer to a low-noise amplifier. Nevertheless, although this new system of coils improved the MCG signal-to-noise ratio, its resolution was not still suitable for real time recordings of the $P$ and $\mathrm{T}$ waves of the cardiac activity.

In 1964, in the Ford Research Labs, the superconducting quantum interference device (SQUID) [3] was invented. This new magnetometer allowed a rapid development of the MCG research [4-20]. After this invention, several SQUID systems for MCG were constructed; for instance, the 67-channels Neuromag system, or the 83 channels PTB-Berlin system. These multi-channel systems can be placed on the whole anterior chest of human subjects to obtain successful MCG recordings of about $50 \mathrm{pT}$.

However, these systems have been mainly used for investigations in humans, and there is scarce information in animal research [16-18]. The aim of the present study was to use a MEMS magnetic field sensor to detect the magnetic flux density emitted by the thorax during respiratory activity of animals in experimental conditions. In a previous report, we performed several tests for the first prototype of this sensor to detect the magnetic flux density of an artificial neuron [21].

\section{Materials and Methods}

\section{Animals}

Experiments were performed using male Sprague-Dawley rats (200-350g). Guidelines contained in 
the National Institutes of Health Guide for the Care and Use of Laboratory Animals (85-23, revised in 1985) and the "Norma Oficial Mexicana NOM-062-ZOO-1999", were strictly followed. Anesthesia was induced with a mixture of ketamine $(100 \mathrm{mg} / \mathrm{kg}$, ip) and xylazine (5.2 $\mathrm{mg} / \mathrm{kg}$, ip). The level of anesthesia was verified throughout the surgery and the whole experiment by monitoring arterial blood pressure and by testing for the lack of withdrawal reflexes and muscle tone. Doses of the ketamine-xylazine mixture were given when necessary. The radial vein was canulated to administer fluids and the carotid artery to monitor blood pressure. A bicarbonate $(100 \mathrm{mM})$ and glucose (5\%) solution was delivered intravenously throughout the experiment at a rate of $5 \mathrm{mlh}^{-1}$. Dextran and saline solutions were given as necessary to maintain blood pressure between 80 and $120 \mathrm{mmHg}$. Body temperature was maintained at $36-37{ }^{\circ} \mathrm{C}$. Rats were placed in a supine position with paws taped to the operating table. With direct visualization of the trachea, an endotracheal tube was inserted and connected to a Harvard rodent volume-cycled ventilator cycling at physiological speeds, with volume sufficient to adequately expand the lungs. The inflow valve was supplied with $100 \%$ oxygen. The sternum bone was removed to obtain an open thoracic cage preparation. We removed tissue to obtain a direct visualization of the heart and the lungs. At the end of the experiment each animal was euthanized with an overdose of pentobarbital. For histological purposes the thorax with heart and lungs was placed in a solution of $10 \%$ formalin.

\section{Electrophysiological recordings}

Shielded custom-made bipolar electrodes were made to reduce artifacts. The electrocardiogram (ECG) signal was obtained through standard three-lead electrodes. Furthermore, other pair of bipolar electrodes was inserted on thoracic muscles to obtain recordings of the electromyogram (EMG) during the respiratory rhythm. The ECG and the thoracic EMG were amplified with GrassP511 amplifiers (Astro-Med, Inc, USA) over a bandwide set between $0.3 \mathrm{~Hz}$ and $10 \mathrm{kHz}$.

\section{Recording of respiratory activity in intact anaesthetized rats}

In other series of 3 experiments rats were anaesthetized with isoflurane via a nose mask. The thorax was keep intact and the MEMS sensor was placed at several positions from the chest surface. In order to detect respiratory activity an electret microphone was coupled to the mask. The microphone and cables were shielded. The output of this amplified and filtered electret signal was send to the input of a window discriminator, which generated trigger pulses. Then the expiratory activity was detected with the window discriminator and it was employed for averaging (32 samples) the signals detected with the MEMS sensor.

\section{The MEMS magnetic field sensor}

Our sensor is a small magnetometer based on MEMS technology developed at the Research Center for Micro and Nanotechnology from the Universidad Veracruzana, Mexico. This research group has publications about this type of MEMS magnetic field sensor [22-24] and some demonstrated applications [21]. The sensor exploits the Lorentz force and has an easy fabrication process, a small size and a high sensitivity. Figure 1A shows an image of the main elements of the proposed sensor. It has a compact resonant structure $(700 \times 600 \times 5 \mu \mathrm{m})$ integrated by an array of silicon beams and uses a piezoresistive sensing system. A signal conditioning system (Figure 1B), together with the sensor, was integrated into a PCB in order to make a digital signal processing, and reduce the electromagnetic noise environment below the signal of interest.
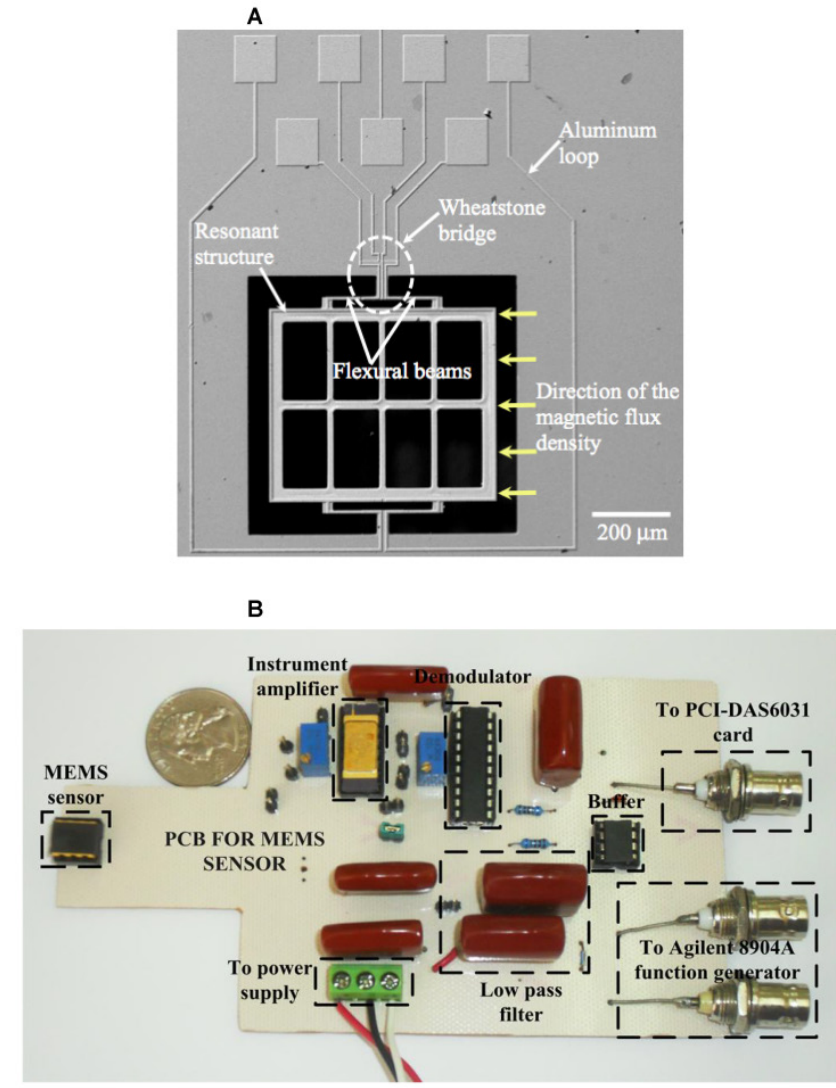

Figure 1. A, Image of a magnetic field sensor based on MEMS technology. B, Image of the signal conditioning system implemented in a PCB for the MEMS sensor. 


\section{Data acquisition}

Data acquisition of the ECG, the thoracic EMG (or the respiratory activity recorded with the electret microphone) and the Magnetic signals recorded with the MEMS sensor was performed at $10 \mathrm{~K} \mathrm{~Hz}$, employing Digidata and Axoscope software (Axon-Instruments). We performed an averaging of the signals by means of a trigger commanded by a window discriminator (WPI, USA). The ECG or the electret microphone recordings were used as the trigger signal. We averaged 32 samples to obtain clear MCG traces. The respiratory magnetogram was also recorded in continuous mode.

\section{Statistical analysis}

Coherence between the rectified an integrated EMG and respiratory magnetogram was calculated. The coherence magnitude of a $95 \%$ confidence interval was also obtained. Data were expressed as mean \pm sd. We used the coherence function of the Matlab software (Mathworks) to perform coherence analysis.

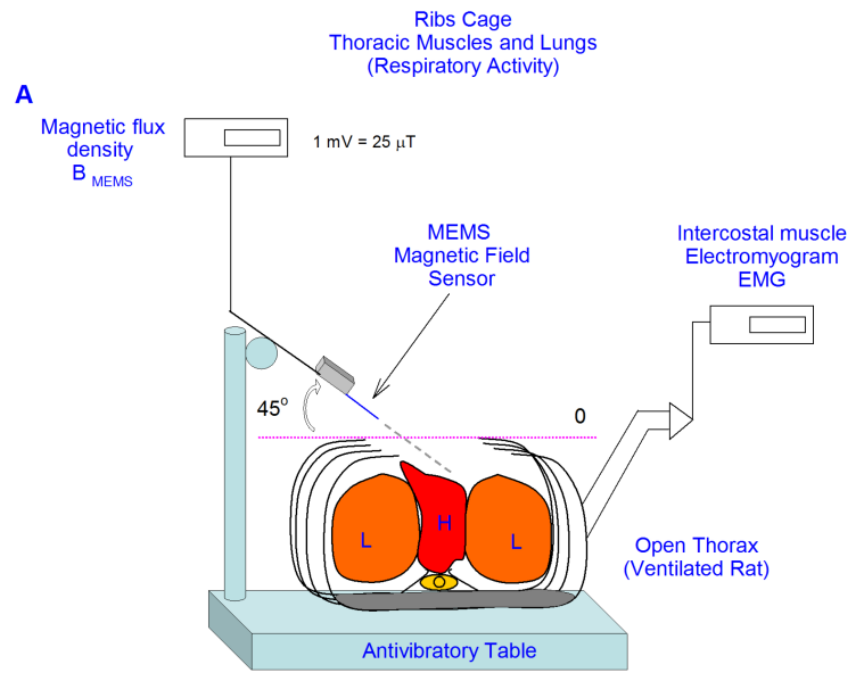

B

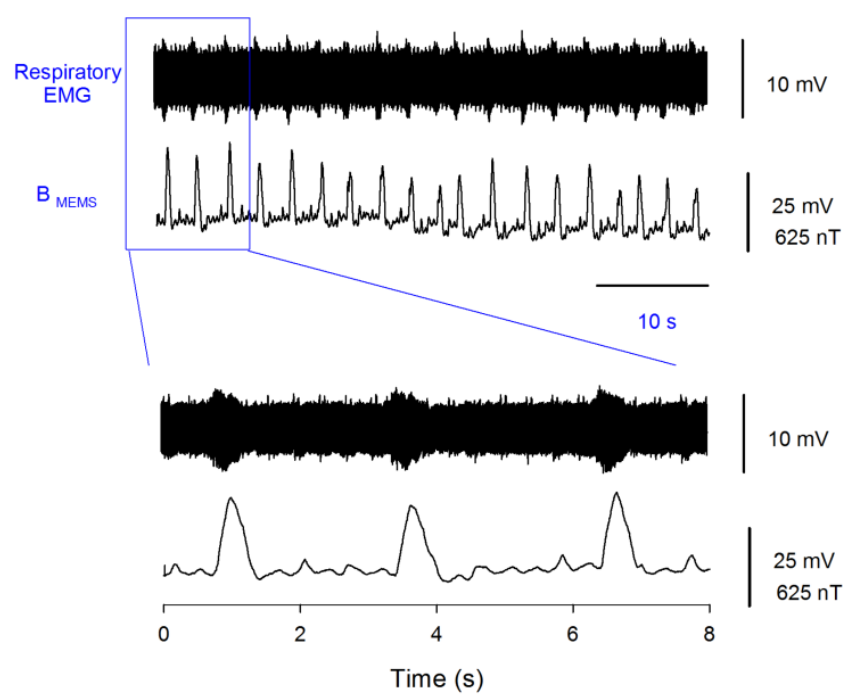

\section{Results}

We performed experiments in 5 anesthetized and ventilated rats. Our experimental paradigm consisted in the recording of the magnetic flux density inside or in the proximity of the rat thoracic cage, which was open at the level of the sternum bone (Figure 2A). In order to find the correct position in which the emitted magnetic field reaches its maximum value we used a micromanipulator to rotate the MEMS sensor throughout different positions and angles. We found a position dependent magnetic flux density, which is probably given by the composition of different magnetic fields generated by the heart, lungs, and thoracic muscles. To avoid artifact movements the MEMS sensor did not touch the rat. In all our experiments the thoracic-muscle electromyogram and the electrocardiogram were also simultaneously recorded, and they served as a reference to compare both magnetic and electric signals generated by activity of heart and thoracic muscles.

\section{C}

D
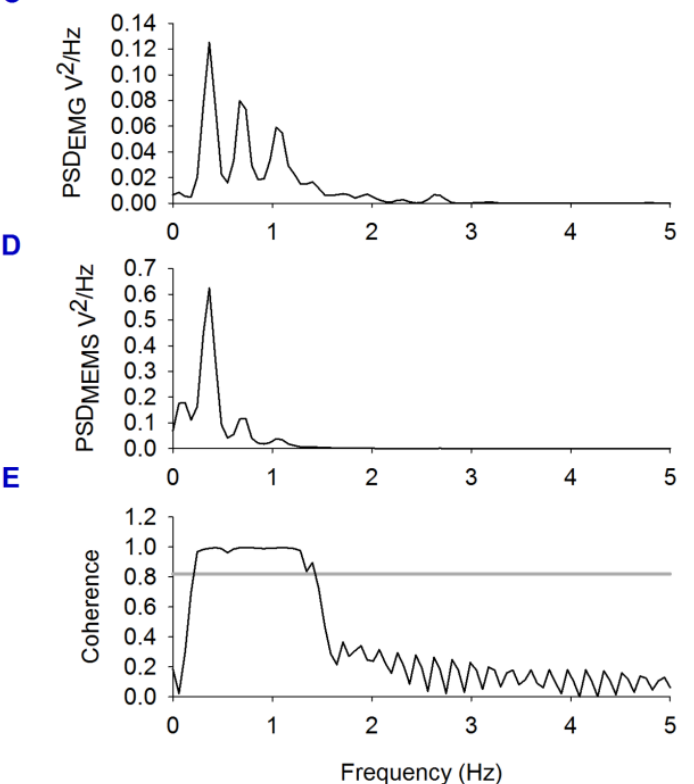

Figure 2. Sensing magnetic flux density of the thoracic cage with a MEMS device during respiratory and cardiac activity. A, Diagram of the experimental arrangement. A MEMS magnetic field sensor was introduced within the open thoracic cage of an anesthetized and ventilated rat. B, Electromyogram of the thoracic muscles and magnetic flux density recordings during the respiratory activity of the rat (respiratory magnetogram). $\mathbf{C}$ and D, Power spectrum density (PSD) calculated from the EMG and from the magnetic flux density, respectively. We found a significant coherence (E) between these rectified and integrated electric and magnetic signals at the respiratory frequency. 
With the MEMS sensor positioned at $0 \mathrm{~mm}$ and $45^{\circ}$, we recorded a strong magnetic flux density produced by muscles of the thorax during respiration, of about $600 \pm 104 \mathrm{nT}$ (mean \pm sd, five rats) (Figure 2B), as well as a MCG of about $98 \pm 49 \mathrm{nT}$ (pink box of Figure 3B). This angle was the optimal because our MEMS sensor detects the maximal component of the magnetic flux density in parallel direction to its resonant structure, as illustrated with yellow arrows in Figure 1A. This is the first evidence that thoracic muscles can produce a strong magnetic flux density during res- piratory activity (i.e., a respiratory magnetogram). Figure 2B shows continuous recordings of the respiratory magnetogram and electromyogram of the thoracic muscles. A significant coherence of about 1 was found between both signals for all the rats; the horizontal line in (Figure 2E) represents the coherence magnitude of a $95 \%$ confidence interval. Similar results as illustrated in Figures 2 and 3 were obtained in 4 other rats. Figure $3 \mathrm{~A}$ illustrates that the MEMS sensor was displaced in different positions inside and outside the thoracic cage.

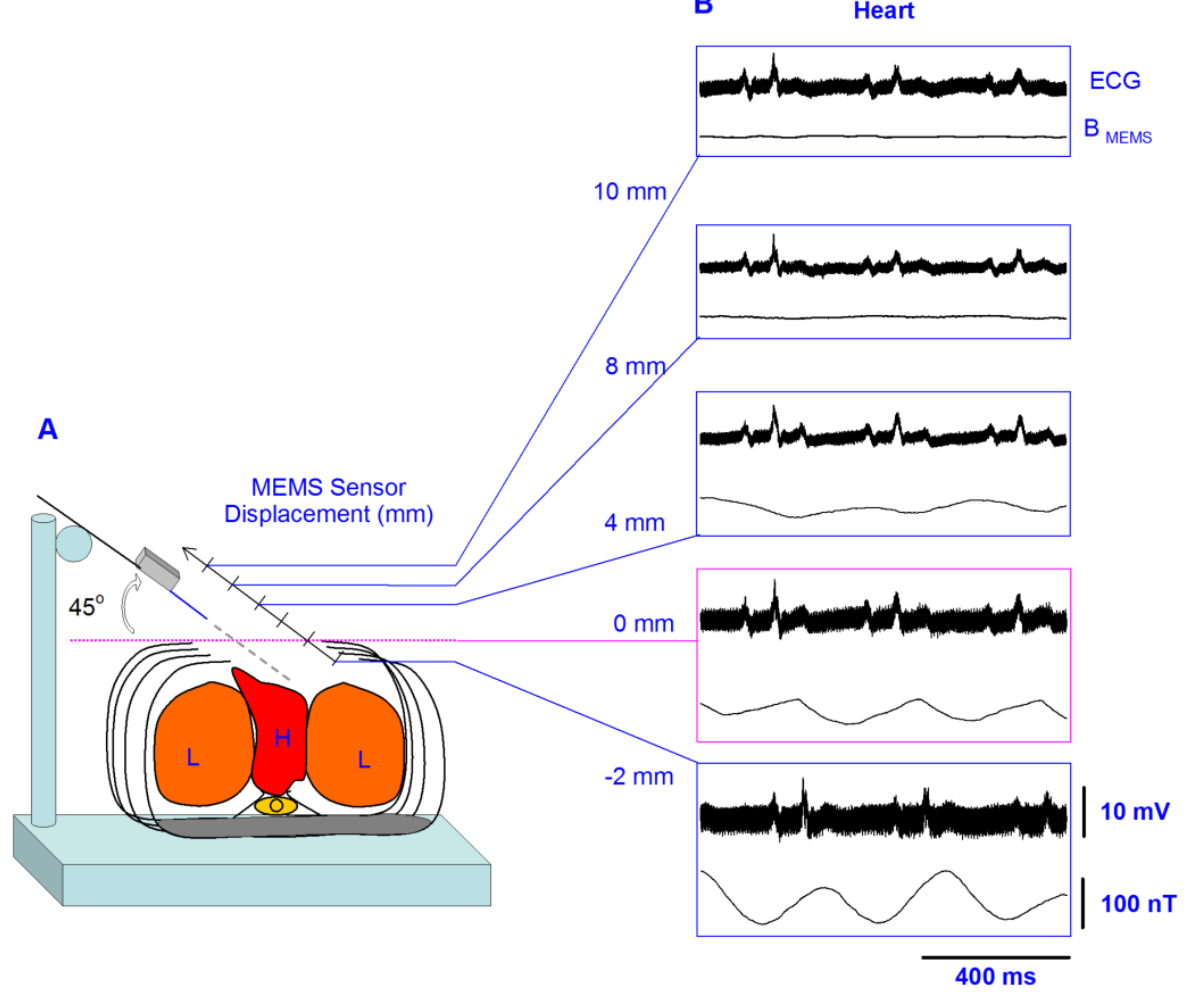

Figure 3. A, Diagram of the experimental arrangement, indicating angle $\left(45^{\circ}\right)$ and positions of the MEMS sensor during the recordings. B, In each box, the upper trace shows the electrocardiogram (ECG) recording; and the lower trace, the magnetic flux density for the respiratory activity (i.e., the respiratory magnetogram $\left.B_{\text {MEMS }}\right)$. The numbers in millimeters $(\mathrm{mm})$ indicate the different positions in which the sensor was placed.

Figure 3B shows the averaged recordings of the electrocardiogram and the MCG obtained with the MEMS sensor in 5 positions. Because the MCG could not be observed in the online recordings we performed and averaging of these magnetic fields by means of an ECG triggering averaging. The recordings in Figure 3B were obtained by averaging over 32 sample measurements. The sensor was positioned at an angle of $45^{\circ}$. Note that at $4 \mathrm{~mm}$ outside the thoracic cage the strength of the magnetic flux density, i.e., the MCG, is close to only about $20 \mathrm{nT}$. However, there is a resonant position at $2 \mathrm{~mm}$ inside the thoracic cage, where the magnetic flux density reaches a maximum value close to $100 \mathrm{nT}$.

We employed the same experimental paradigm and the same recording angle of $45^{\circ}$ as in Figure 3, but for the respiratory magnetogram in three other rats. Figure 4B illustrates averaged recordings (32 samples) of this respiratory magnetogram for one rat. Figure 4B shows that these signals exhibit their maximal amplitude for positions close to the thorax (about $-2 \mathrm{~mm}$ to $4 \mathrm{~mm}$ ). We calculated the grand average of the respiratory-magnetogram amplitude for three rats and we obtained graphs of this grand average versus the recording position (Figure 4D). We found an inverse relationship between the magnitude of this respiratory magnetogram and the recording position.

Furthermore, we employed the same experimental paradigm, angle of $45^{\circ}$ and positions, as in Figure $4 \mathrm{~B}$, but in three other intact rats. Figure $4 \mathrm{C}$ shows averaged recordings (32 samples) of this respiratory magnetogram for one intact rat. We found a 
maximal magnitude of this respiratory magnetogram for positions close to the thorax as illustrated in Figure 4C. Furthermore, as illustrated in Figure 4E, we also found an inverse relationship between the magnitude of this respiratory magnetogram and the recording position (results obtained from the grand average of the respiratory-magnetogram amplitudes of three intact rats).

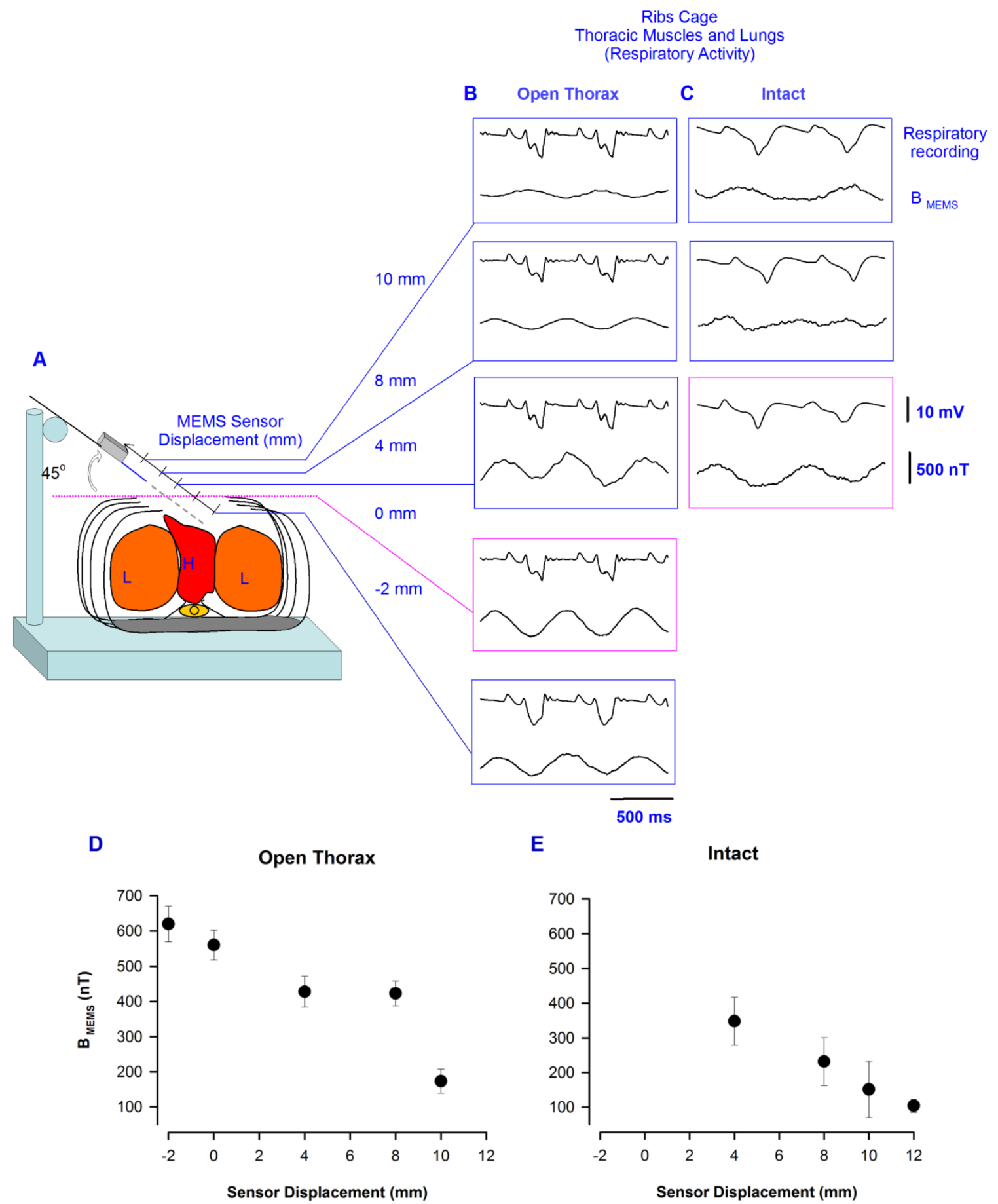

Figure 4. A, Diagram of the experimental arrangement, indicating angle $\left(45^{\circ}\right)$ and positions of the MEMS sensor during the recordings. B, In each box, the upper trace shows the respiratory activity detected with the electret microphone; and the lower trace, the magnetic flux density for the respiratory activity (i.e., the respiratory magnetogram $B_{M E M S}$ ). C. The same as B, but for an intact rat. The numbers in millimeters $(\mathrm{mm})$ indicate the different positions in which the sensor was placed. $\mathbf{D}$ and $\mathbf{E}$, Grand average of the respiratory magnetogram versus the sensor position, for three rats with the open thorax and three other intact rats, as indicated (mean \pm sd). 


\section{Discussion}

We recorded a strong and rhythmic respiratory magnetogram of about $600 \mathrm{nT}$ by means of a MEMS sensor. In all the ventilated rats, we observed that after an overdose of ketamine the respiratory magnetogram was completely abolished, thus suggesting that the magnetic signal detected with the MEMS sensor was not produced by a movement artifact due to the rodent ventilation system. We suggest that a synergistic contraction of the thoracic muscles mainly contributes to the emission of the magnetic field that is recorded with the MEMS device.

In our experiments, with the open thorax of the rat, we also recorded a MCG of about $100 \mathrm{nT}$ which is about 2,000 or 10,000 times stronger than the typical MCG detected on the chest of human subjects (50 pT) or rats $(10 \mathrm{pT})$ with the SQUID magnetic detector, respectively. Discrepancies in the magnitude of these magnetic flux densities could be explained for differences in the animal preparation employed (i.e., open thorax, in our case versus intact thorax in other studies). An alternative explanation could be the close proximity to the heart of our MEMS sensor, inside the thoracic cage with an optimal angle, regarding the horizontal plane of the chest. Other explanation could be the method of ECG triggering averaging of the MCG, which exclude uncorrelated signals from the MCG.

Fluctuations in strength of the respiratory magnetogram could be employed to analyze the respiratory dynamics in different physiological aspects. In this context, our findings could attract the attention of physicians, physiologists, biologists and biomedical researchers.

\section{Acknowledgements}

This work was supported by the following grants: FORDECyT-CONACyT 115976 (AH) and CONACyT-84605-and-95111 (PJGR) to develop the MEMS sensor. CONACyT-100028 to purchase a Gaussmeter (EG). Cátedra Marcos Moshisky, PROMEP-PIFI-BUAP and CONACyT F1-62610-and153583 (EM). PROMEP-Red: "Instrumentación-de-Sensores-para-aplicaciones-de-Fisiología-y-Bi omedicina", México. The authors would like to thank Pablo Linares for technical assistance in the experiments and John Reid for proof reading the English manuscript.

\section{Competing Interests}

The authors have declared that no competing interest exists.

\section{References}

1. Baule G, McFee R. Detection of the magnetic field of the heart. Am Heart J. 1963; 66: 95-96.

2. Lekkala JO, Malmivuo JAV. Simultaneous measurement of the magnetic heart vector components with unipositional lead system. In: Erne SN, Hahlbohm HD, Lubbig H, ed. Biomagnetism, Berlin: de Gruyter; 1980: 319-326.

3. Zimmerman JE, Theine P, Harding JT. Design and operation of stable rf-biased superconducting point-contact quantum devices, etc. J Appl Phys. 1970; 41:1572-1580.

4. Cohen D. Measurements of the Magnetic Fields Produced by the Human Heart, Brain and Lungs. IEEE Trans. Magn. 1970; 2: 694-700.

5. Cohen D, Edelsack E, Zimmerman J. Magnetocardiograms taken inside a shielded room with a superconducting point contact magnetometer. Appl Phys Lett. 1970; 16: 278-280.

6. Cohen D, Edelsack E, Zimmerman J. Magnetocardiograms Taken Inside a Shielded Room with a Superconducting Point Contact Magnetometer. Appl Phys Lett. 1970; 16: 278-280.

7. Cohen, D. et al. MEG versus EEG localization test using implanted sources in the human brain. Ann Neurol. 1990; 28: 811-817.

8. Lekkala JO, Mallmivuo JAV. Multiplexed SQUID vector magnetometer for biomagnetic research. J Phys E Sci Instrum. 1984; 17: 504

9. Koch, H. Recent advances in magnetocardiography. J Electrocardiol. 2004; 37: 117.

10. Li Z, Xue-Min Z, Li-Hua Z, Xu-Guang H, Yu-Feng R, Geng-Hua C, Qian-Sheng Y, Ji F. An economical magnetocardiogram system based on high-T SQUIDs. Chinese Phys Lett. 2006; 23: 2319-2322.

11. Lee YH, Yu KK, Kim JM, Kwon H, Kim K, Park YK. 64-channel second-order axial gradiometer system based on DROS for magnetocardiogram in a thin shielded room. Physica C. 2006; 468: 1942-1945.

12. Lee YK, Kim K, Kwon H, Lee YH. Signal amplitude ratio of magnetocardiogram between sensor assembly and chest surface. IEEE T Magn. 2009; 45: 2886-2889.

13. Lim HK, Chung N, Ko YG, Lee YH, Park YK. Magnetocardiogram difference between healthy subjects and ischemic heart disease patients. IEEE T Magn. 2009; 5: 2890-2893.

14. Kang CS, Lee YH, Kim J, Yu KK, Kim JM, Kwon H, Kim IS, Park YK, Lim HK, Lee SG. Comparison of magnetocardiograms measured using different SQUID pickup coil configuration. IEEE T Appl Supercon. 2009; 17: 835-838.

15. Janawadkar MP, Radhakrishnan TS, Gireesan K, Parasakthi C, Sengottuvel S, Patel R, Sundar CS, Baldev RAJ. SQUID-based measurement of biomagnetic fields. Curr Sci 2010; 99: 36-45

16. Kim IS, Ahn S, Lee CH, Lee YH. Development of a mouse biomagnetic measurement system by using a high-T (c) SQUID magnetometer. IEEE T Appl Supercon. 2011; 21: 493-496.

17. Kim IS, Kwon H, Ahn S, Song JH. Measurement of rat magnetocardiograms by using a high-T (c) SQUID magnetometer system. IEEE T Appl Supercon. 2011; 21: 497-500.

18. Michael LH, Ballantyne CM, Zachariah JP, Gould KE, Pocius JS, Taffet GE, Hartley CS, Pham TT, Daniel SL, Funk E, Entman ML. Myocardial infarction and remodelin in mice: effect of reperfusion. Am J Physiol-Heart C. 1999; 277: H660-H668.

19. Maslennikov YV. Magnetocardiographic diagnostic complexes based on the MAG-SKAN SQUIDs. J Commun Technol El. 2011; 56: 991-999.

20. Leithaeuser B, Jung F, Park JW. Magnetocardiography in clinical cardiology. Status quo and future applications. Postepy kardiologii I JNEJ. 2011; 7: 215-222.

21. Tapia JA, Herrera-May AL, Garcia-Ramirez PJ, Martinez-Castillo J, Figueras E, Flores A, Manjarrez E. Sensing magnetic flux density of artificial neurons with a MEMS device. Biomed Microdevices. 2011; 13: 303-313.

22. Herrera-May AL, Garcia-Ramirez PJ, Aguilera-Cortes LA, Martinez-Castillo J, Sauceda-Carvajal A, Garcia-Gonzalez L, Figueras-Costa E. A resonant magnetic field microsensor with high quality factor at atmospheric pressure. J Micromech Microeng. 2009; 19: 01516.

23. Herrera-May AL, Garcia-Ramirez PJ, Aguilera-Cortes LA, Figueras E, Martinez-Castillo J, Manjarrez E, Sauceda A, Garcia-Gonzalez L, Juarez-Aguirre R. Mechanical design and characterization of a resonant magnetic field microsensor with linear response and high resolution. Sensor Actuat A-Phys. 2011; 165: 399-409.

24. Herrera-May AL, Aguilera-Cortes LA, Garcia-Ramirez PJ, Manjarrez E. Resonant Magnetic Field Sensors Based on MEMS Technology. Sensors. 2009; 9: 7785-7813. 